\title{
Which atoms are where? Which fields are around?
}

\author{
Hannes Lichte
}

Institute of Applied Physics, Dresden University, D 01062 Dresden, Germany, www.triebenberg.de

Electron holography has been developed a powerful technique with remarkable advantages over conventional imaging methods: It provides both amplitude and phase, linear and zero-loss, it allows a-posteriori correction of all imaginable coherent aberrations, and it offers all the means for a complete wave optical analysis. Holography is intrinsically quantitative hence a unique tool for measuring at nm dimensions.

On one hand, large fields of view of about $1 \mu \mathrm{m}$ allow measurements of mesoscopic microfields in and around objects, e.g. magnetization distribution, potentials in semiconductors and polarisation in ferroelectrics at a potential sensitivity of better than $0.1 \mathrm{~V}$. On the other hand, atomic resolution of about $0.1 \mathrm{~nm}$ has been reached, which not only allows to delineate "where is an atom", but also to answer the question "which atom is where" from quantitative evaluation of the atomic phase shift differing with the atomic number. However, large fields of view and atomic resolution cannot be combined arbitrarily with the need of accuracy. To give an example: a Porsche may have a top speed of $270 \mathrm{~km} / \mathrm{h}$, and it may be driven around a curve as narrow as $9 \mathrm{~m}$ in diameter - however not at the same time; these two figures of merit are incompatible with each other. Likewise, in electron holography, the two figures of merit "lateral resolution of $0.1 \mathrm{~nm}$ " and "phase sensitivity $2 \pi / 1000$ " are incompatible.

First of all, there are technical restrictions by the pixel-number of the available CCD-cameras, which strictly connect the field of view and the reachable lateral resolution. Due to the needs of holography, every object pixel has to be covered by 3 hologram fringes, each of which has to be sampled by 4 CCD-pixels. Therefore, 12 CCD-pixels define one reconstructed pixel hence the strong need for high pixel CCD-cameras, which will solve this problem. However, from the point of view of measuring, there are much more fundamental limitations between field of view, lateral resolution and signal resolution, which stem from Quantum Noise. These are outlined in the following.

Accuracy and sensitivity of phase measurements can be described by the phase detection limit $\delta \varphi$, which sets a lower limit for the phase difference detectable between two adjacent pixels in the reconstructed phase image. $\delta \varphi$ depends on the quality of the hologram, on the properties of the object, and on the desirable lateral resolution

$$
\delta \varphi=\text { NoiseFigure } \cdot \operatorname{snr} \cdot \frac{2 q_{\text {rec }} w}{V_{\text {inel }}} .
$$

$q_{\text {rec }}$ is the spatial frequency reconstructed from a hologram of width $w$, and $V_{\text {inel }}$ the contrast reduction by inelastic interaction with the object; snr means the signal/noise-number, at which $\delta \varphi$ is to be measured. The hologram may be characterized by 


$$
\text { NoiseFigure }=\frac{\sqrt{2 \pi}}{|\mu| V_{\text {inst }} M T F \sqrt{\left(I_{c o h}(\mu) / e\right) \varepsilon t \cdot D Q E}}
$$

with the total current $I_{c o h}(\mu)=-\ln (|\mu|) \frac{B}{k^{2}}$ available at the degree of coherence $\mu$ from a source of brightness $B$ and wave number $k ; V_{\text {inst }}$ means contrast reduction by instabilities, $\varepsilon$ is the ellipticity of illumination, and $t$ exposure time; $M T F$ and $D Q E$ describe the CCD-camera. For example, reasonable data of our microscopes yield NoiseFigure $\approx 4 \cdot 10^{-4}$.

Fig. 1 shows the relation between the number of reconstructed pixels $N_{\text {rec }}=2 q_{\text {rec }} w$ and the achievable phase detection limit $\delta \varphi$. Expectedly, $\delta \varphi$ increases (deteriorates) proportional to $N_{\text {rec }}$, i.e. the "information volume" $\frac{\delta \varphi}{N_{\text {rec }}}$ is constant for any given hologram: either one can optimize phase sensitivity, or lateral resolution, but not both at the same time.

Fig. 2 shows the corresponding plot for voltage resolution at dopant profiling, translated by means of $\varphi=\sigma \cdot V_{\text {projected }}(\sigma:=$ interaction constant); optimum object thickness of twice the mean free path for inelastic interaction is assumed, which leads to inelastic contrast damping by $V_{\text {inel }}=1 / e$. In order to obtain a voltage resolution of $0.1 \mathrm{~V}$, one has to restrict to about 55 reconstructed pixels. This in turn means that, to reach the desirable $0.1 \mathrm{~V}$ at a lateral resolution of $1 \mathrm{~nm}$, the field of view, i.e. the width of the hologram, has to be restricted to $50 \mathrm{~nm}$. Similar considerations hold for the corresponding problems at atomic resolution.

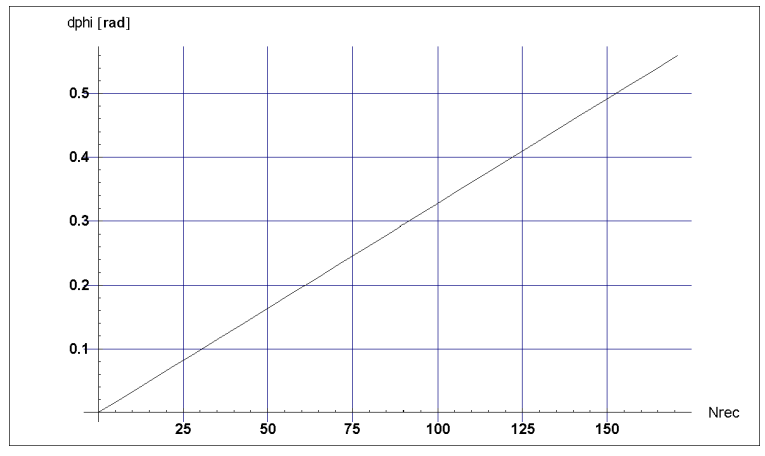

Fig.1 Phase detection limit for $s n r=3$, $V_{\text {inel }}=1 / \mathrm{e}$ and NoiseFigure $=4 \cdot 10^{-4}$.

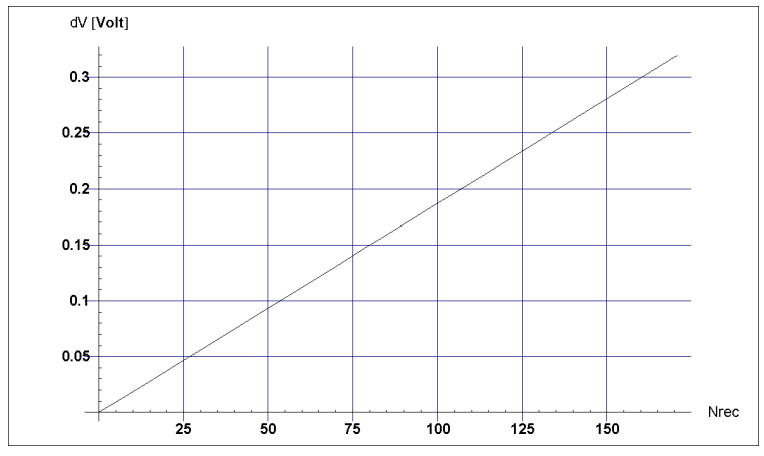

Fig.2 Voltage resolution for an object of optimum thickness vs. reconstructed pixel number 